\title{
Evolución de los sistemas de juego en el fútbol: Una revisión sistemática
}

\author{
Evolution of playing systems in soccer: A systematic review
}

Lic. Frank Gabriel Tapia Jara. ${ }^{1}$

Recibido: 01-03-2021 / Revisado: 18-03-2021 /Aceptado: 25-03-2021/ Publicado: 05-04-2021

\begin{abstract}
.
DOI: https://doi.org/10.33262/cienciadigital.v5i2.1620

Introduction. Soccer is a team sport, turning out to be the most practiced in the world. With the historical passing of the game systems used in this sport have varied from an ultra-offensive system to the present time that variants of systems are used in the same game. Objective. Systematize the theories related to the evolution of game systems in football and their relationship with the optimization of sports performance. Methodology. The research was carried out using a descriptive, non-experimental methodology, supported by the use of theoretical methods and documentary review; allowing the RSL (Systematic Literature Review) to be carried out in a time range between 2000 and 2020. To obtain quality information, a logical order was followed, which included the planning, analysis and evaluation of the results and findings mainly found in databases and high impact scientific articles preceded by the establishment of the inclusion and exclusion criteria determined, under the determination of the search criteria: game systems "," soccer "," sport " evolution. Results. 1. The importance, relevance and timeliness of the investigated topic is determined. 2. The variability and complexity of the game systems in football are not only related to the position that the players occupy within the field, it is a complex, deep process, with multiple views and dimensions. Tactical learning through different game strategies requires a solid cognitive development on the part of the participating footballers in such a way that it facilitates the motor application of what has been learned. Conclusions. The importance and timeliness of the subject is systematized, showing the need to continue deepening it, in order to provide studies that allow to show more intentionally the relationship between the different game systems and optimal sports performance.
\end{abstract}

\footnotetext{
${ }^{1}$ Universidad Católica del Ecuador, Ecuador, fgtapia@uce.edu.ec, ORCID: https://orcid.org/0000-00032351-8331
} 
Keywords: game systems, soccer, sports performance, evolution

\section{Resumen.}

Introducción. El fútbol es un deporte de equipo resultando ser el más practicado a nivel mundial. Con el decursar histórico los sistemas de juego utilizados en este deporte han variado desde un sistema ultraofensivo hasta la actualidad que se utilizan variantes de sistemas en un mismo partido. Objetivo. Sistematizar las teorías relacionadas con la evolución de los sistemas de juego en el fútbol y su relación con la optimización del rendimiento deportivo. Metodología. La investigación se realizó mediante una metodología descriptiva, no experimental, apoyados en el empleo de métodos teóricos y la revisión documental; permitiendo efectuar la RSL (Revisión Sistemática de Literatura) en un rango de tiempo comprendido entre el 2000 y el 2020. Para obtener información de calidad se siguió un orden lógico, que incluyó la planificación, análisis y valoración de los resultados y hallazgos encontrados principalmente en bases de datos y artículos científicos de alto impacto precedidos por el establecimiento de los criterios de inclusión y exclusión determinados, bajo la determinación de los criterios de búsquedas: sistemas de juego", "fútbol”, "deporte”, "evolución”. Resultados. 1. Se determina la importancia, pertinencia y actualidad del tema investigado. 2. La variabilidad y complejidad de los sistemas de juegos en el fútbol, no solo están relacionados con la posición que ocupan los jugadores dentro del terreno, se trata de un proceso complejo, profundo, de múltiples miradas y dimensiones.3. El aprendizaje táctico mediante las diferentes estrategias de juegos requiere de un desarrollo cognitivo sólido por parte de los futbolistas participantes de modo tal que facilite la aplicación motriz de lo aprendido. Conclusiones. Se sistematiza la importancia y actualidad del tema, quedando evidenciado la necesidad de continuar profundizando en el mismo, en función de aportar estudios que permitan evidenciar con mayor intencionalidad la relación entre los diferentes sistemas de juegos y el óptimo desempeño deportivo.

Palabras clave: sistemas de juego, fútbol, desempeño deportivo, evolución

\section{Introducción.}

El fútbol es un deporte de equipo que se ha convertido en el más practicado a nivel mundial, en los diferentes niveles, debido a su sencilla reglamentación y a los pocos requerimientos materiales que se necesita para su práctica. No obstante, una significativa importancia radica en su táctica y sistemas de juego aplicados, las cuales han tenido cambios continuos perfeccionándose con el paso del tiempo.

Con el decursar histórico los sistemas de juego utilizados en este deporte han variado desde un sistema ultraofensivo hasta la actualidad que se utilizan variantes de sistemas en un mismo partido. Atendido a lo señalado y ante la diversidad de criterios existentes en relación con la evolución de los sistema de juegos y su incidencia en el óptimo desempeño 
deportivo la investigación que se presenta tiene como objetivo: Sistematizar las teorías relacionadas con la evolución de los sistemas de juego en el fútbol y su relación con la optimización del rendimiento deportivo.

\section{Un acercamiento conceptual.}

Son varios los autores que se han referido al respecto, entre los que podemos citar a Morales \& Murillo (2013), quienes señalan que el fútbol es un deporte que se sostiene en un sistema táctico o sistema de juego que facilita la organización colectiva de los jugadores dentro del campo, garantizando una adecuada ocupación racional de los jugadores en el terreno de juego.

Las definiciones del sistema de juego son variadas, por lo que diferentes autores emplean criterios diferenciados, que nos permiten a su vez evidenciar la evolución que han tenido estos sistemas en el tiempo.

En este orden encontramos a autores como: Tassara (1967), Csanadi (1969), Teodorescu (1984), Ferrández (1996), quienes afirman que la elección del sistema de juego depende directamente de las características individuales de los jugadores y no cambia de partido a partido.

Maturana, citado por Cuadrado (2001), plantea que "el sistema de juego es simplemente un punto de partida, después que las circunstancias del partido varían las posiciones iniciales", en función a lo largo del partido de si el equipo tiene la posición del balón.

Por su parte autores como (Zeeb, 1994; Yagüe \& Sánchez, 2005), son del criterio que al momento de elegir un sistema de juego, debe tenerse en consideración los "máximos espacios de juego posible para cada uno de los jugadores, permitiéndoles cierta creatividad en las situaciones del juego así como cambios de posiciones y funciones dentro de la estructura del equipo".

De igual manera, Floro (2005) explica que cualquier sistema de juego se fundamenta en "el modo de atacar y de defender y la táctica es atacar y defender, o sea es el método de juego, o sea la manera de realizar un ataque, una defensa y un contraataque, partiendo de unas posiciones. En fin el sistema es el modo de ejecutar el juego".

En esta dirección, el sistema de juego del fútbol se ha ido transformando, evolucionando de una concepción inicial altamente ofensiva a una actual donde existe un predominio de la defensa por sobre la ofensiva, incidiendo de manera determinante en la organización táctica estructural de los equipos de futbol, la cual está determinada por su propia dimensión estática y dinámica.

Por otra parte, Vales (2012), define los sistemas de juego como la manera táctica en que están dispuestos los patrones básicos de organización y funcionamiento de un equipo, en 
donde se determina, por una parte la actitud de los jugadores y su filosofía de juego a realizar al encontrase con el rival y, por otra parte, se regularizan tanto las posiciones fundamentales y radios de acción de los jugadores, como las tareas o funciones encomendadas a éstos, ya sea en términos individuales (puesto específico), grupales (líneas/sectores de campo) y colectivos (equipo).

Este mismo autor plantea que sus principales características son: su estabilidad, su dinamismo, su flexibilidad, solidaridad, especificidad y operatividad, así como, que los sistemas de juego están organizados internamente en tres componentes: el Componente Conceptual que marca la filosofía de juego, el Componente Formal, que establece la relación del equipo con el espacio de juego, o sea el modo en que se colocan los jugadores en el campo de juego y el Componente Funcional, referido a la aplicación de los diferentes métodos de juego y a la distribución de las tareas tácticas entre los jugadores.

En referencia a ello, Lovrincevich (2002), destaca la importancia que dentro del sistema de juego reviste tanto la dimensión estática como la dinámica, indicando que la primera está referida al sistema de juego o esquema táctico que está representado por el modo de colocación de los jugadores en el campo de juego, ya sea 4-3-3, 5-4-1, 4-4-2, y otros, la que conforma la formación que establecerá el orden y los equilibrios de las diferentes zonas del terreno de juego, sirviendo como área de referencia y de partida para la realización por los jugadores, de los desplazamientos relativos así como para la coordinación de las diferentes acciones individuales y colectivas.

Según Casteló (1999), la dimensión dinámica está referida a las distintas tareas y misiones tácticas que previamente han sido distribuidas entre los jugadores que conforman el equipo.

No obstante, a todo lo señalado existen varios elementos que influyen en la determinación y elección de un sistema de juego, en relación a ello concordamos con Díaz (2017a), al formular que las diferentes tácticas de juego dependen directamente de las habilidades técnicas de los jugadores, las capacidades físicas de los mismos, el estado psicológico de los deportistas, la calidad y manera de jugar del equipo rival así como del estilo que el entrenador proyecte aplicar para obtener sus objetivos.

De igual manera, este mismo autor en relación a ello enunció que los sistemas de juego aplicados serán más eficientes y eficaces en función de las características físicas y técnico- tácticas de los jugadores y su relación con la posición que tengan dentro del esquema táctico y la responsabilidad que enfrenten dentro del campo de juego.

En esta misma línea Mercé (2006), resaltó algo importante a tener en cuenta, señalando que la forma de desarrollar cada partido de futbol y las variantes que pueden aplicarse en cada sistema de juego de un equipo está en función del comportamiento del equipo contrario, de cómo se encuentre el marcador del juego, así como de factores que no 
pueden preverse, (lesiones, expulsión, entre otros aspectos), que son aspectos que fundamentan el componente táctico-estratégico del equipo.

Independientemente de todo lo destacado y en esta misma dirección compartimos el criterio de Guedea, et al. (2019), al señalar que esto no implica que exista un sistema de juego perfecto, se coincide que todos los sistemas tácticos tienen fortalezas y debilidades.

En relación a ello, autores como, Mercé et al. (2008), han afirmado que el sistema de juego no hay que considerarlo como un fin en sí mismo, este debe ser flexible y debe estar al servicio del equipo, sin implicar en ningún caso su detrimento, o limitar el desarrollo de muchos jugadores que lo integran, precisamente porque todos tienen necesidades diferentes al resto.

Al respecto estamos de acuerdo con Díaz (2017b), al afirmar que según su criterio no hay formaciones de fútbol buenas o malas, ofensivas o defensivas, y lo que realmente determina el éxito o el fracaso de un partido de fútbol, son las interpretaciones que hacen los propios jugadores de esa disposición, sumado con la calidad técnica que tengan los mismos que les permita improvisar jugadas y el nivel de acierto en la toma de decisiones durante el juego y en los tiros a puerta.

Lo expuesto con anterioridad nos reafirma que sin un sistema de juego sería imposible a un equipo de fútbol avanzar en su propio desarrollo funcional del juego, en relación a ello Sánchez (2014), ha asentado que la falta de un sistema de juego indicaría un equipo sin referencias posicionales de los jugadores, las cuales son de gran utilidad para facilitar el desarrollo del juego y citaba que el destacado entrenador Carlos Queiroz, afirmaba que la distribución de los jugadores en el campo de juego, siempre tenía influencia sobre el desarrollo del juego que realizaría el equipo dado que su disposición en el terreno ofrece racionalidad al modo de jugar del conjunto.

En relación a ello, García-Calvo et al. (2002), opinó que el técnico Pep Guardiola decía que en el fútbol había que partir de un orden y de una idea de juego predeterminada, o sea, se parte de la necesidad de crear un escenario de actuación, en el que los jugadores deben moverse para realizar las acciones que se van sucediendo durante el juego. En esa línea, planteó que la clave es que el equipo se encuentre lo suficiente organizado para que los esfuerzos que se realicen por los jugadores no generen un desequilibrio en el propio equipo.

Es por estas razones importantes considerar que independientemente que los sistemas de juego se han venido transformando en el decursar del tiempo, ello no implica que la diferencia de esos cambios se produzca en su totalidad en un tiempo determinado. En investigaciones realizadas por diversos autores entre los que tenemos a Castellano, et al. (2008), se ha corroborado que de los datos obtenidos en el diagnóstico de la evolución del juego en los 3 mundiales $(1998,2002,2006)$, únicamente el 1\% de la variancia explicada nos hace decantarnos con argumentos, a partir de las variables o facetas 
tomadas en la configuración del modelo, indicándose que el fútbol no ha variado significativamente.

En este orden de idea algunos autores como: Díaz, et al. (2011), han realizado investigaciones en las que han demostrado que los sistemas de juego, desde el punto de vista de la organización posicional, han evolucionado hacia un sistema defensivo. Ello se evidencia en que en los inicios del fútbol los preparadores y entrenadores se preocupaban por buscar ventajas numéricas en las zonas de ataque, dándole una gran importancia al juego ofensivo de los equipos. Con el paso de los años, y con las innovaciones reglamentarias introducidas, especialmente la referida a la regla del fuera de juego, la prioridad ha sido implantar un sistema de juego creando una superioridad numérica en las zonas defensivas y creando oportunidades en las zonas más propicias para hacer gol.

No obstante, los sistemas de juego no constituyen un concepto que deba imponerse, debe ser estudiado y practicado en función de las potencialidades técnico-tácticas de cada equipo, en este orden autores y a la vez entrenadores de futbol, como Kordon, et al. (2007), han afirmado que un entrenador debe tener una claridad de la idea de juego y utilizar un sistema de juego con diferentes variantes según las fortalezas del equipo, las debilidades, el rival, el tipo de campo de juego, el marcador del partido y muchos otros factores, que no se pueden ser soslayados, pero principalmente en base a las características físicas y técnicas de los jugadores.

En esta dirección se han realizado diversos estudios dentro de los cuales se destacan los aportes de: González, et al. (2015), quienes han afirmado que en los últimos años ha aumentado el interés hacia esta línea de investigación, principalmente en el proceso de enseñanza-aprendizaje en jóvenes jugadores desde la perspectiva táctica de los sistemas de juego.

En esta misma línea, debe enfatizarse en que los sistemas de juegos propiamente funcionan de manera compleja, por lo que recaba de los jugadores un conocimiento profundo de la táctica de juego. Al respecto, autores como Morales \& Murillo (2013), han afirmado que los modelos de juego en el fútbol son más que un sistema de distribución de jugadores, dado que por su dinámica presentan una elevada complejidad, requiriendo de la organización de los futbolistas que interaccionan, mediante reglas de acción intrínsecas al mismo. Compartimos con los autores precedentes en que el modelo de juego que se implementa conceptualiza la organización desde su totalidad, aunando el complejo ensamblaje de tendencias, funcionalidades y características que se produce entre todas las posiciones ocupadas por los jugadores de un equipo.

Es por ello que los sistemas de juego han seguido transformándose y mejorando para ser más competitivos, principalmente de la mano de talentosos entrenadores que han partido de muchos sistemas creados y utilizados durante muchas décadas y han aplicado conceptos teóricos avanzados logrando una consolidación del juego de sus respectivos 
equipos, alcanzado grandes éxitos como campeones en diversas competiciones de liga, continentales y mundiales.

Otros autores como Moreno (2016), han realizado estudios de investigación en los cuales se ha evidenciado que las características morfológicas, fisiológicas, psicológicas y técnico-tácticas que forman parte del perfil de un jugador de futbol, han ido evolucionando de manera paralela a la concepción y sistemas de juego empleados.

Es por ello que se hace necesario, considerar la necesidad de actualizar de forma periódica el estudio de los sistemas de juego en el fútbol, tomando en cuenta lo más destacado de cada uno de estos sistemas y a su vez para que sirva de punto de partida para la creación de nuevos sistemas de juego.

La presente investigación servirá para que los entrenadores que aún se encuentran en el trabajo empírico se informen sobre fortalezas y amenazas de cada sistema y poder incorporar a sus equipos el esquema de juego ideal que encaje con las características de sus jugadores.

Por otra parte, dada la generalidad de la práctica del fútbol y la constante elevación de su nivel competitivo, conlleva a que a la vez que se constituya en uno de los deportes que más recursos económicos invierte en la investigación para mejorar la competitividad, ello implica la realización de continuas investigaciones sobre los diversos aspectos del mismo.

No obstante a ello, aún existen algunos aspectos del juego en los que no se han realizado estudios profundos y otros que carecen de actualización, por lo cual es importante y necesario que se actualicen los estudios, en este caso que nos ocupa, el referido al tema sobre los sistemas de juego en el fútbol que no se ha encontrado bibliografía actualizada en este tema.

\subsection{Clasificación de los sistemas de juego. Sus Fortalezas y debilidades.}

Los sistemas de juego, como se ha planteado con anterioridad representan la manera en que están dispuestos inicialmente los futbolistas en el campo de juego, desde el mismo principio del futbol como deporte, este aspecto ha constituido la piedra angular de su accionar y como el propio deporte ha sufrido constantes variaciones en su desarrollo.

Basándonos en lo expuesto, compartimos criterios con Agulló (2020), al plantear que los sistemas de juego en el fútbol presentan una concepción general que gira alrededor de la posición inicial y teórica de los jugadores en el terreno de juego, que permite en función de ello establecer una clasificación.

De manera general los sistemas de juego están propensos a una clasificación en función del concepto de elaboración, en relación a este aspecto, varios autores tales como: Castelo (1999) y Mercè (2006) lo subdividen en: Sistemas de juego basados en la amplitud y aquellos basados en la ocupación racional del campo de juego. 
Los sistemas de juego basados en la amplitud, se distinguen por su amplitud ofensiva y poca profundidad defensiva cuando el rival posee el balón. Tiene como finalidad la ocupación eficiente de los espacios, para adueñarse del balón y posteriormente crear situaciones de desequilibrio en el área del rival.

Dentro de estos sistemas tenemos a:

$\checkmark$ Sistemas de juego de posición: 1-3-4-3 / 1- 4-3-3, el cual busca mantener la posesión del balón en todo momento y en especial en la fase de ataque.

$\checkmark$ Sistema de ocupación e incorporación: 1-3-5-2 / 1-4-5-1, buscan la profundidad ofensiva, incorporando jugadores desde segunda línea para ocupar los espacios generados detrás de las líneas del equipo contrario.

$\checkmark$ Sistemas de juego mixtos: 1-3-5-2 / 1-4-5-1, tiene como fin crear y ejecutar un juego más equilibrado, que le permite tener una mayor ventaja en determinadas zonas del terreno de juego.

En el caso de los sistemas de juego basados en la ocupación racional del campo de juego, están determinados por la óptima ocupación racional del espacio del terreno, presentando líneas horizontales y verticales, que refleja una evidente profundidad defensiva. Tales son los casos de:

$\checkmark$ Sistemas de juego en el plano transversal: 1-4-3-3 / 1-4-4-2, presentan líneas horizontales muy pobladas, priorizando la posesión del balón a la profundidad.

$\checkmark$ Sistemas de juego en el plano longitudinal: 1-3-4-3 / 1-4-2-4 / 1-3-2-5. Se posiciona a los jugadores de manera que enlazan las posiciones de una línea con la otra.

Teniendo en cuenta lo anterior, distintos autores se refieren a que las más básicas distribuciones de los sistemas de juego presentan como principal característica, el equilibrado posicionamiento de los jugadores en el campo de juego, con su base más amplia en la defensa, reforzando la teoría de que un buen equipo se construye con una buena defensa.

En esta dirección Díaz (2017a), concibe que el sistema que más se adecúa a este sistema de juego es el 1-4-3-2-1, utilizado por reconocidos técnicos que han obtenido varios triunfos en competiciones a todos los niveles. Otros sistemas de juego básico y que más se utilizan actualmente se relacionan a continuación.

Sistema de juego fútbol 1442

Es el sistema idóneo para desarrollar un estilo de juego directo o de contragolpe. 
Figura No 1 Sistema de juego fútbol 1442

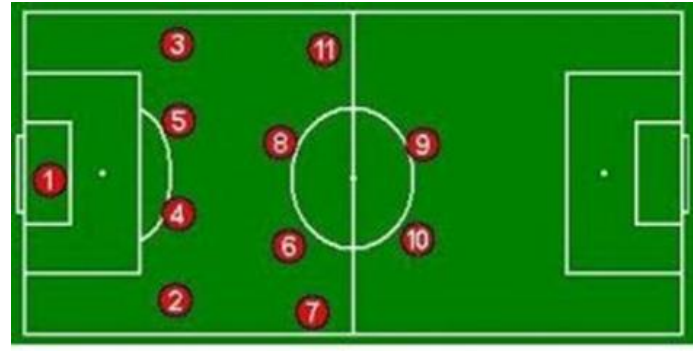

FORMACIÓN 1-4-4-2

Fortalezas del sistema de juego 1-4-4-2

Este sistema de juego, permite a los atacantes primarios avanzar sin tener que esperar el apoyo de los centro campistas, por lo que el medio campo y la defensa no necesitan retrasar sus intentos de llevar el balón a posiciones avanzadas, favoreciendo a que los mejores delanteros sean capaces de enfrentarse a una gran variedad de situaciones de juego con un mínimo apoyo del medio campo.

\section{Debilidades del sistema de juego 1-4-4-2}

Las mismas están referenciadas a la previsibilidad y la rigidez del sistema de juego, así como a la presión que se ejerce sobre los centros campistas para atacar y defender de manera constante.

Sistema de juego fútbol 1-4-4-2 Rombo.

Esta variante del sistema, está indicado para elevar el nivel de posesión del balón, ya que aporta superioridad numérica de jugadores en el área del centro del campo. Pudiera parecer que se renuncia a las bandas pero no es así. Contar con laterales ofensivos estos ocupará los carriles por fuera.

Figura No 2. Sistema de juego fútbol 1-4-4-2 Rombo.

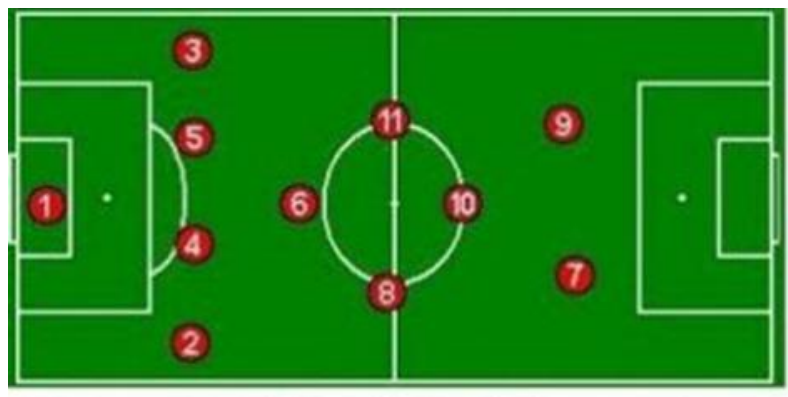

FORMACIÓN 1-4-4-2 ROMBO

Sistema de juego fútbol 1-4-3-3. 
Este sistema es sinónimo de fútbol de ataque, de posesión continua del balón. Una de sus características más importantes es la amplitud del terreno de juego que se abarca a través de sus extremos. Dos jugadores interiores que deben tener una gran condición física, jugadores área-área, deben tener llegada en segundas jugadas pero a su vez deben ayudar en defensa.

Figura No 3. Sistema de juego fútbol 1-4-3-3.

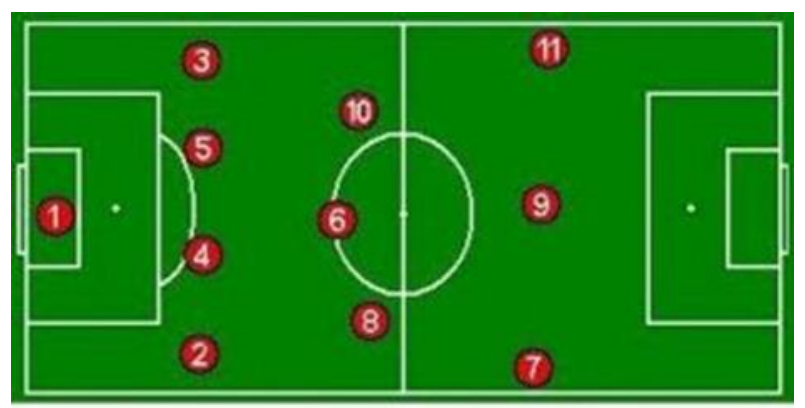

FORMACIÓN 1-4-3-3

\section{Fortalezas del sistema de juego 1-4-3-3}

Permite dominar la posesión del medio campo con sus tres jugadores contra equipos que juegan sólo dos.

Permite anular la amenaza que representan los laterales de un adversario, jugando con dos delanteros muy avanzados, limitando las opciones de ataque del oponente y permitiendo la posesión del balón en el mediocampo y disminuyendo sustancialmente las oportunidades de contraataque.

Debilidades del sistema de juego 1-4-3-3

Para su implementación con éxito, se debe contar con jugadores que tengan la capacidad de pensar y actuar de manera rápida en lo que respecta a su posicionamiento y la distribución del balón.

El delantero central tiene la gran responsabilidad de tomar posesión regular del balón y hacerlo llegar a los otros dos delanteros atacantes. Esta acción no puede realizarse por muchos delanteros centrales cuando se encuentran a una defensa de élite.

Sistema de juego fútbol 1-3-5-2.

Este sistema es una de las mejores formaciones ofensivas de juego, no obstante puede ser clasificado como defensivo u ofensivo, dependiendo de las situaciones del juego. Por lo regular se utilizan a tres jugadores centrales y dos laterales.

Fortalezas del sistema de juego 1-3-5-2 
Cuándo los extremos están en repliegue se convierte en un 1-5-3-2 de ahí la parte ultra defensiva del mismo.

\section{Debilidades del sistema de juego 1-3-5-2}

En la fase ofensiva, la línea defensiva se reduce a tres jugadores, debilitándose la misma.

Sistema de juego fútbol 1-4-2-3-1

En este sistema la pelota se traslada a través de triángulos, siendo más efectivo para derribar a los oponentes que hacer avanzar la misma en líneas rectas.

Figura No 4. Sistema de juego fútbol 1-4-2-3-1.

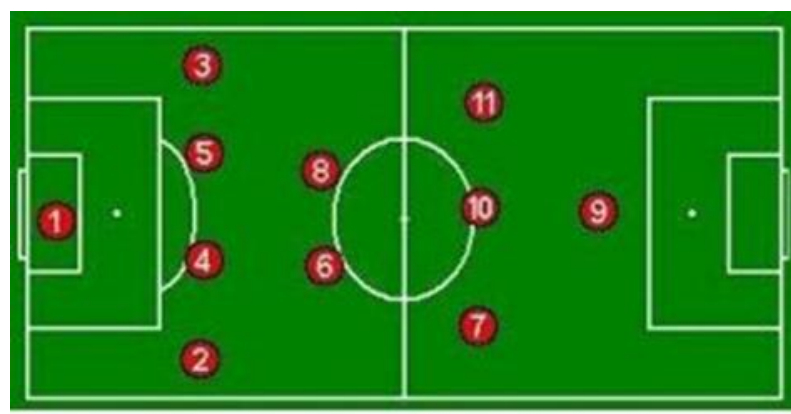

FORMACIÓN 1-4-2-3-1

Fortalezas del sistema de juego 1-4-2-3-1

La posición de los dos mediocampistas en relación a los otros tres más avanzados permite generar opciones de pase del balón, eliminando el problema del juego recto o lateral de otros sistemas.

El número de jugadores atacantes potenciales permite que alguno de ellos se mueva a mayor profundidad para responder ante una oportunidad repentina de contraataque.

Además, con tantos jugadores listos y disponibles para pasar el balón hacia adelante, al delantero en esta formación se le suelen dar muchas oportunidades de marcar.

\section{Debilidades del sistema de juego 1-4-2-3-1}

El esfuerzo físico que tienen que realizar los centrocampistas y delanteros puede generar problemas en la defensa, dado que algunos equipos contrarios comienzan los ataques desde el núcleo de sus defensas.

Lo anterior remarca la presión que realmente existirá sobre los jugadores atacantes, que les exige que los mismos jueguen con una exactitud de tiempo que les permita moverse 
de un extremo a otro del campo, de manera que se impida a los mediocampistas y laterales del adversario recuperar sus posiciones e iniciar ataques.

Sistema de juego fútbol 1-3-4-3.

Este sistema es también de carácter muy ofensivo y de posesión continua del balón, abarcando una amplia parte del terreno de juego.

Figura No 5. Sistema de juego fútbol 1-3-4-3.

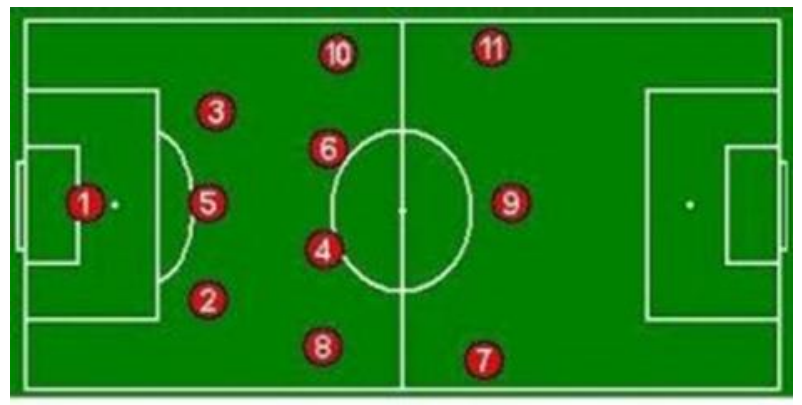

FORMACIÓN 1-3-4-3

\section{Fortalezas del sistema de juego 1-3-4-3}

Permite el dominio de la posesión del medio campo con sus cuatro jugadores contra equipos que juegan sólo tres o dos.

El número de jugadores atacantes potenciales permite que alguno de ellos se mueva a mayor profundidad para responder ante una oportunidad repentina de contraataque.

\section{$\underline{\text { Debilidades del sistema de juego 1-3-4-3 }}$}

En la fase ofensiva, la línea defensiva se reduce a tres jugadores, debilitándose la misma.

Los centro campistas deben contar con el físico adecuado para participar apoyando el ataque de los delanteros y retroceder para apoyar, ante cualquier situación a la defensa.

\section{Metodología.}

La investigación se realizó mediante una metodología descriptiva, no experimental, apoyados en el empleo de métodos teóricos, tales como: el histórico-lógico, el analíticosintético, inductivo-deductivo y la revisión documental; permitiendo así realizar la RSL (Revisión Sistemática de Literatura). Para obtener información de calidad se siguió un orden lógico, que incluyó la planificación, análisis y valoración de los resultados y hallazgos encontrados principalmente en bases de datos y artículos científicos de alto impacto. La consulta de la literatura precedente contempló un rango de tiempo entre el 2000 y el 2020, incluyendo la valoración de los aportes realizados en Tesis de Doctorado, Maestría y Licenciatura en relación con el tema objeto de estudio. 
El control de la calidad y el rigor científico del trabajo presentado estuvo precedido por el establecimiento de los criterios de inclusión y exclusión determinados, bajo los criterios de búsqueda: sistemas de juego", "fútbol”, “deporte”, "evolución”,

Criterios de inclusión

1. Tipo de estudios: Estudios de revisiones teóricas o experimentales que permitan sistematizar las teorías precursoras en relación con la evolución de los sistemas de juegos en el fútbol y su significación en el rendimiento deportivo.

2. Tipo de participantes: investigadores, entrenadores, docentes, deportistas relacionados con el fútbol.

3. Tipo de resultados: Programas de entrenamiento, Metodologías, modelos, que sistematicen o contemplen experiencias relacionadas con el tema objeto de estudio

4. Tesis de Doctorado o Maestría realizadas en los últimos años en Universidades Internacionales o Nacionales sobre el tema de investigación.

Criterios de exclusión

1. Estudios o investigaciones realizadas en un rango de tiempo mayor al contemplado en la investigación.

2. Artículo en idioma diferente del español o inglés.

3. Otros estudios que por su tema o resultados no aporten elementos reveladores para nuestra investigación.

\section{Resultados}

El análisis y valoración de las bases de datos consultadas comprendió un rango entre el 2000 y el 2020, consultándose un total de 25 fuentes relacionadas con el tema objeto de estudio, determinándose dentro de estas 7 investigaciones potenciales para el estudio y sistematización de las teorías relacionadas con la evolución de los sistemas de juegos en el fútbol y su trascendencia en el rendimiento deportivo.

Especial significación adquirieron los aportes realizados por Sánchez, (2014) en su tesis de Doctorado, al analizar el desarrollo del juego de fútbol 11, desde la óptica de los sistemas complejos. En sus aportes el autor enfatiza en la necesidad de analizar más profundamente cómo va evolucionando el equipo durante un partido, el rol que toma cada jugador y el peso que tiene dentro del equipo, según su distribución y la posición que ocupa éste sobre el terreno de juego.

Emanado de la revisión bibliográfica realizada se aprecia además que dentro de los autores que más han aportado al análisis realizado se destacan: Casteló (1999), Mercé (2006), Sánchez (2014), Díaz (2017a, 2017b), Guedea, Nájera, Núñez, Candía \& Gastélum (2019), los cuales destacan en su trabajo la necesidad de tener una mirada 
amplia en relación con los sistema de juegos dentro del fútbol, acentuando que no sólo se trata de tener en cuenta la posición de los jugadores dentro del terreno, sino también valorar otros elementos más complejos y de especial alcance como es por ejemplo los señalados por Moreno (2016), al referirse a las características morfológicas, fisiológicas, psicológicas y técnico-tácticas que forman parte del perfil de un jugador de fútbol.

A ello se une las contribuciones de Agulló (2020), al plantear que los sistemas de juego en el fútbol presentan una concepción general que gira alrededor de la posición inicial y teórica de los jugadores en el terreno de juego. En este orden se coincide con Floro (2005), en que los sistemas de juego del fútbol se han ido transformando, evolucionando de una concepción inicial altamente ofensiva a una actual donde existe un predominio de la defensa por sobre la ofensiva, incidiendo de manera determinante en la organización táctica estructural de los equipos de fútbol

De todo lo anterior se coincide con lo señalado por Morales \& Murillo (2013), al afirmar que los modelos de juego en el fútbol son más que un sistema de distribución de jugadores. Al respecto es criterio del investigador que es importante ver estos en toda su dimensión y profundidad, debido a que los sistemas de juegos funcionan de manera compleja, de ahí la importancia de determinar previamente qué línea seguir, cómo y cuándo actuar dentro del juego. Cabe recalcar que cada jugador tiene un importante papel a cumplir dentro del partido, cada uno desempeña disímiles funciones pero todos trabajan por un mismo objetivo: obtener la victoria.

Ello nos permite asumir que el éxito estaría en la determinación previa de la estrategia de juego a seguir y la comprensión que se debe lograr de esta por parte de cada jugador en correspondencia con sus funciones dentro del juego. Se considera asimismo que debido a la complejidad de los diferentes sistemas de juegos, características de los participantes $\mathrm{y}$ avances logrados, se hace muy necesario que los jugadores posean un conocimiento profundo de la táctica del juego a seguir, comprendiendo sus tendencias, funcionalidad, características y posiciones dentro del equipo, así también deben poseer conocimientos profundos del equipo contrario.

Encontrar un equilibrio en esto ha venido constituyendo sin lugar a dudas un gran reto para la comunidad deportiva y científica en general; precisamente porque si bien es cierto que con el transcurrir histórico se han realizado diferentes investigaciones relacionadas con los sistemas de juego en el fútbol, donde han aflorado posicionamientos teóricos de gran valía y actualidad, los cuales han posibilitado una evolución positiva del tema, aún resultan insuficientes tanto en el contexto ecuatoriano como internacional el desarrollo de investigaciones que permitan encontrar alternativas y aportar soluciones encaminadas a establecer estrategias de juegos más completas, dinámicas y personalizadas, que estén en correspondencia con las propias individualidades de los jugadores participantes.

Por otra parte se requiere enfatizar y profundizar en el desarrollo de investigaciones que permitan establecer con mayor claridad la relación que existe entre los sistemas de juego 
y su significación en el rendimiento deportivo; máxime si tenemos en cuenta que ambas variables están estrechamente relacionadas, constituyendo una unidad dialéctica esencial a considerar para la mejora de los resultados deportivos.

Obsérvese la Tabla No1, donde se muestran los resultados del análisis realizado en cuanto a las investigaciones potenciales directos para la presente investigación.

Tabla No 1 Resultado de Investigaciones potenciales directos para la investigación
Autorías,
Base de Datos
Intervención-
Conclusiones
Titulo y año

Kordon P, Perrone E, Pochetino J (2007) - Evolución de los sistemas de juegos: historia y actualidad
Castellano J, Perea A, Hernández A (2008). Análisis de la evolución del fútbol a lo largo de los mundiales. https://prof.webcindario. com/evolucion de los sistemas de juego.pdf.

Psicothema 2008. Vol. 20, no 4, pp. 928-932. ISSN $0214 \quad$ - 9915 CODEN PSOTEG. www.psicothema.com. Universidad de Oviedo. España
La evolución de los sistemas de juego del fútbol es un área para estudiar detalladamente.

Los 3 sistemas de juego que consideramos para el análisis son el 4-4-2, que predomina principalmente en los equipos de primer nivel europeos y en el fútbol argentino, el 35-2 que predominó en Corea-Japón 2002 y el 4-5-1 que fue la base de Alemania 2006.

Esta obsesión por pensar primero en la defensa antes que en el ataque, por tener más jugadores defensivos que ofensivos, hizo que fuera descendiendo progresivamente la cantidad de goles en los Torneos.

El estudio que se presenta continúa una línea de investigación iniciada hace ya algunos años sobre el análisis de la generalizabilidad y componentes de variancia aplicados a la acción de juego en fútbol,

que nos ha permitido situar la investigación en un punto crucial. El
Evolución de los sistemas de juego: A medida que fueron pasando los años, el número de defensores fue creciendo mientras el número de delanteros disminuyó.

Cantidad de goles en los Mundiales: Como resultado de esa obsesión por tener más defensores que delanteros, por defender antes que por atacar, el número de goles por partido disminuyó notoriamente.

Un entrenador debe tener una idea de juego y utilizar un sistema de juego con diferentes variantes según las fortalezas, las debilidades, el rival, la localía, el resultado y muchos factores más que no se pueden pasar por alto. Pero principalmente en base a los Jugadores.

Se pudo interpretar que en los tres mundiales analizados (1998- 20022006) se ha

notado cierta diferencia en la forma que los equipos tienen de jugaren función de si van ganando, empatando o perdiendo $\mathrm{y}$ consiguen marcar $\mathrm{o}$ encajar un gol. Además cuando se considera en interacción. Además lo que nos hace interpretar 
trabajo es el resultado de la observación, codificación y registro de los contextos de interacción

desarrollados por las diferentes selecciones nacionales que han participado en la fase final de los mundiales de Francia'98, Japón y Corea'02

Alemania'06, sumando un total de 58 partidos.

Díaz R, Álamo H, XIV Seminario

Del Toro G, Internacional y II Hernández J (2011)- Latinoamericano de Estudio y análisis de Praxiología Motriz: los sistemas de juego en fútbol.

e
Educación Física y contextos críticos. Departamento Educación Facultad de de Humanidades y Ciencias de la Educación Universidad Nacional de La Plata. Sitio web http://seminprax.fahce.un lp.edu.ar/. La Plata, 12 al 15 de octubre de 2011 ISBN 978-950-34-08209

Morales Y, Murillo E (2013)- Origen y evolución de los sistemas tácticos del futbol
Proyecto de grado válido parcialmente para optar al título de Profesional en Ciencias del Deporte. Universidad del Valle. Instituto Educación y Pedagogía Profesional en Ciencias del Deporte. Santiago de Cali.
Los sistemas de juego nos permiten esa organización colectiva de los jugadores dentro del campo y garantizan una adecuada ocupación racional del terreno de juego, necesaria para cumplir con las acciones de juego de las fases mencionadas anteriormente.

De allí la necesidad de conocer más profundamente la importancia y las características de los principales sistemas de juego, desde su origen, su proceso evolutivo $\mathrm{y}$ de desarrollo, y su que en los tres mundiales analizados la variabilidad del juego ha sido más bien escasa, por no decir prácticamente nula.

De este estudio preliminar, podemos hacer dos consideraciones que nos parecen de gran importancia. La primera que nos consideramos necesario abordar el estudio y análisis de la acción de juego, con una nueva perspectiva en la que predominen los aspectos informacionales y de significación práxica, por encima de los mecánicos o de ejecución, especialmente de las conductas motrices o subroles estratégicos motores. Esto implicará además que hagamos uso de manera prioritaria de una metodología observacional sistemática. Al realizar el análisis de las ventajas y desventajas de los sistemas tácticos utilizados el futbol actual, se plantea que a la enseñanza de la táctica le corresponde un aprendizaje de tipo cognitivo que tiene su importancia en el campo de aplicación motriz. El jugador debe conocer $\mathrm{y}$ entender la

estructura funcional y la dinámica del juego, de lo contrario, actuará sin comprender lo que hace; la desventaja de este hecho es que los conocimientos adquiridos, de manera indiferenciada y por azar, se convierten en 
aplicabilidad en el automatismos que se

Sánchez J (2014)- Tesis de Doctorado. Análisis del Universidad de las desarrollo del juego de fútbol 11, desde la óptica de los sistemas complejos.

González S, García L, Contreras O (2015). Evolución de la toma de decisiones y la habilidad técnica en fútbol. ISSN: 1577 0354.
Palmas de Gran Canaria. España.

Revista Internacional de Medicina y Ciencias de la Actividad Física y el Deporte vol. 15 (59) pp. 467-487.

Http://cdeporte.rediris.es/ revista/revista59/artevolu cion613.htm futbol actual, así como también la necesidad de definir y explicar de forma clara y sencilla, la gran cantidad de términos y conceptos de esta disciplina, para su mejor comprensión

utilizan de forma rígida $\mathrm{y}$ no reflexiva, conllevando a cometer muchos errores y al estancamiento del pensamiento táctico.

En todos los datos obtenidos se puede comprobar el efecto positivo que tiene la enseñanza teórico / práctica proposicional sobre la comprensión de la lógica interna del juego del fútbol y el comportamiento táctico.

Este trabajo de investigación trata de combinar el análisis clásico de este tipo de modalidad deportiva con las propuestas basadas en la teoría de los sistemas complejos no lineales. Para ello se han seleccionado cuatro ámbitos de estudio, que tratan de abordar el fenómeno del fútbol desde una visión más general a otra más específica.

Este artículo presenta como se desarrolla la toma de decisiones y la habilidad técnica en jugadores con alto nivel de pericia desde los 6-7 a 13-14 años.

Los resultados se basan en el análisis inferencial correlacional. La evolución del rendimiento de juego se orienta desde el ataque hasta la defensa. Se han encontrado diferencias significativas en las variables entre las cuatro categorías de formación estudiadas, especialmente en el principio táctico de progresar hacia la portería contraria y en el desmarque, así como en el marcaje y la ayuda en defensa.
Aplicando una serie de herramientas, podemos analizar más detalladamente y más profundamente cómo va evolucionando el equipo durante un partido, el rol que toma cada jugador y el peso que tiene dentro del equipo, según su distribución y la posición que ocupa éste sobre el terreno de juego.

La integración de las aportaciones científicas en el campo pedagógico debería ser visible en los campos de fútbol con jugadores en formación. De este estudio, se extrae información valiosa para el entrenador en relación a aspectos tales como la superación del egocentrismo en las primeras etapas, la alternancia en la enseñanza entre ataque y defensa, el aumento del número de jugadores en el juego competitivo de referencia. Dado que este estudio está basado en un ataque libre y una defensa individual, serían necesarios más estudios que analizasen otros tipos de ataque o de defensa, por ejemplo las defensas zonales en fútbol son muy 
Moreno A (2016)- Tesis de fin de grado. En este estudio "Evolución del Universidad de León. trataremos de hacer fútbol profesional y México.

su repercusión en

los requerimientos

físicos de los jugadores".

una reflexión evolutiva de este deporte, analizando de la manera más objetiva posible las principales frecuentes y habría que estudiarlas. dimensiones que han contribuido a innovar constantemente la esencia de esta modalidad tales como el perfil del entrenador y de sus jugadores, las dimensiones tácticas derivadas de los mismos, los avances en las investigaciones científicas consecuentemente de los sistemas de entrenamiento, la importancia del "Match análisis", también relacionando estos conceptos.
Se ha percibido una clara tendencia evolutiva hacia la utilización de sistemas de juego que favorecen y promueven un planteamiento técnicotáctico de maniobra, y priorizando cada vez más el aspecto defensivo en detrimento del ofensivo. Esta evolución en la concepción del juego ha ido generando una mayor concentración de jugadores en el medio campo.

Cada vez se le da mayor libertad a la figura del entrenador y de su cuerpo técnico a la hora de tomar decisiones de ámbito táctico-estratégico, físico e incluso en el ámbito económico (fichajes), teniendo más oportunidades que en épocas anteriores de adaptar el juego del equipo acorde a su propia concepción del juego.

La evolución en los sistemas de "Match analysis" ha favorecido la proliferación de numerosos estudios tácticos, que nos permiten una inmensa malgama de dimensiones de análisis. Todo esto puede ayudar a visualizar aspectos tácticos y combinativos a mejorar (analizando el propio equipo), o bien a potenciar los que se consideren oportunos (analizando debilidades del rival).

Una vez revisada las literaturas precedentes y expuestos los criterios generales es preciso resumir cuatro ideas fundamentales que encierran en sí los resultados obtenidos:

1. La RSL (Revisión Sistemática de Literatura), permitió determinar que el tema relacionado con la evolución de los sistema de juegos en el fútbol y su trascendencia en el rendimiento deportivo se ha venido tratando en diferentes 
investigaciones científicas realizadas desde hace varios años, sin embargo dada la propia evolución que se ha logrado tener en las estrategias de juego, el comportamiento y característica de los jugadores, la variabilidad de las técnicas y recursos a emplear, entre otros aspectos, hacen que este tema sea de mucho interés, pertinencia y actualidad científica.

2. El otro elemento a considerar y en el cual se debe enfatizar es que la variabilidad y complejidad de los sistemas de juegos en el fútbol, no solo están relacionados con la posición que ocupan los jugadores dentro del terreno, se trata de un proceso complejo, profundo, de múltiples miradas y dimensiones que requiere de atención permanente para poder alcanzar la optimización del rendimiento deportivo de los jugadores participantes.

3. A esto se debe agregar que todo proceso de aprendizaje táctico mediante las diferentes estrategias de juegos requiere de un desarrollo cognitivo sólido por parte de los futbolistas participantes de modo tal que facilite la aplicación motriz de lo aprendido, es por ello fundamental que el jugador conozca y comprenda la estructura funcional y la dinámica del juego a desarrollar.

4. La optimización del rendimiento deportivo está estrechamente relacionada con la estrategia de juego a seguir.

\section{Conclusiones}

Según el análisis y revisión de la literatura precedente se concluye:

- Se sistematiza la importancia y actualidad del tema, quedando evidenciado la necesidad de continuar profundizando en el mismo, en función de aportar estudios que permitan evidenciar con mayor intencionalidad la relación entre los diferentes sistemas de juegos y el óptimo desempeño deportivo.

- La evolución de los sistemas de juegos en el fútbol y su trascendencia en el rendimiento deportivo demuestran que se trata de un proceso altamente complejo, dinámico de múltiples miradas y dimensiones, que transita desde el desarrollo cognitivo que garantice el conocimiento y comprensión de la estrategia a seguir por parte de cada jugador hasta la aplicación en la práctica de lo aprendido.

- La evolución se marca en la existencia de sistemas de juegos ultraofensivo hasta la actualidad que se utilizan variantes de sistemas en un mismo partido.

\section{Referencias Bibliográficas}

Agulló, C., (2020). Los Sistemas de Juego en Fútbol (Tesis de Grado). Universidad da Coruña, España.

Castelo, J., (1999). Fútbol Estructura y dinámica del juego. Barcelona: Inde.

Castellano, J., Perea, A., Hernández, A., (2008). Análisis de la evolución del fútbol a lo largo de los mundiales. Psicothema, 20 (4), 928-932. 
Cuadrado, P., (2001). Entrevista táctica a Francisco "Pacho" Maturana. Revista Training Fútbol, (69), 8-15.

Csanádi, A. (1969). El Fútbol: técnica, táctica y sistema de juego. Preparación física Entrenamiento. Barcelona: Planeta.

Díaz, R., Álamo, H., Del Toro, G. y Hernández, J. (2011). Estudio y análisis de los sistemas de juego en fútbol. XIV Seminario Internacional y II Latinoamericano de Praxiología Motriz: Educación Física y contextos críticos. Departamento de Educación Física Facultad de Humanidades y Ciencias de la Educación Universidad Nacional de La Plata. Recuperado de: http://seminprax.fahce.unlp.edu.ar/

Díaz, M. (2017a). El mundo del entrenador de fútbol, entrenamiento, técnica, táctica y estrategia. Recuperado de: https://bit.ly/3r0Fb82

Díaz, M. (2017b). Formaciones de Futbol. Recuperado de: https://bit.ly/38ROwJe

Ferrández, J. (1996). Fútbol entrenamiento físico basado en la táctica y la estrategia. Madrid: Gymnos.

Floro, B., (2005). Bases en el desarrollo del sistema defensivo zonal. Revista Training Fútbol, (115), 18-21.

García-Calvo, J.A., Benjamín, Z.E., López-Marco, J., Pérez-Caminero, L., Amavisca, J. E., Sánchez-Domínguez, C. y Guardiola, J. (2002). La táctica desde el punto de vista del jugador. Revista Training Fútbol (81), 16-23.

González, S., García, L., Contreras, O., (2015). Evolución de la toma de decisiones y la habilidad técnica en fútbol. Revista Internacional de Medicina y Ciencias de la Actividad Física y el Deporte. 15 (59), 467-487. https://doi.org/10.15366/rimcafd2015.59.005

Guedea J, Nájera R, Núñez O, Candía R. y Gastélum G (2019). Sistemas tácticos y resultados de competición del Mundial de Fútbol Asociación de Rusia 2018. Retos: nuevas tendencias en educación física, deporte y recreación, (36), 503-509.

Kordon, P., Perrone, E. y Pochetino, J. (2007). Evolución de los sistemas de juegos: historia y actualidad. Recuperado de: https://bit.ly/3rYfAOD

Lovrincevich, C., (2002). Análisis de la evolución de los sistemas de juego en el fútbol. Revista Digital 8 (53), 1-12. 
Mercé, J., (2006). Apuntes de la asignatura de táctica y sistemas de juego. Curso de Técnicos Deportivos- Nivel II. Sevilla, España.

Mercé, J., Ródenas, L., y Dómenech, C., (2008). Fútbol los sistemas de juego: Sistema 1.4.2-3.1. Sevilla: Wanceulen Editorial Deportiva

Morales, Y. y Murillo, E. (2013). Origen y evolución de los sistemas tácticos en el futbol. Proyecto de grado válido parcialmente para optar al título de Profesional en Ciencias del Deporte (Tesis de Grado). Universidad del Valle, Santiago de Cali, Colombia.

Moreno, A., (2016). Evolución del fútbol profesional y su repercusión en los requerimientos físicos de los jugadores (Tesis de Grado). Universidad de León, México.

Sánchez, J., (2014). Análisis del desarrollo del juego de fútbol 11, desde la óptica de los sistemas complejos (Tesis Doctoral). Universidad de las Palmas de Gran Canaria, España.

Tassara, H., (1967). Fútbol simplemente. San José: Antonio Lehman.

Teodorescu, L., (1984). Problemas de la teoría y metodología nos desportos colectivos. Lisboa: Livros horizonte.

Vales, A., (2012). Fútbol: Del análisis del juego a la edición de informes técnicos. Madrid: MC sports.

Yagüe, J., \& Sánchez, J., (2005)- Propuesta para la organización de actividades que faciliten el rendimiento del futbolista: el calentamiento para la competición. Revista Cuadernos Técnicos, (33), 33-43.

Zeeb G., (1994) Manual de Entrenamiento de Fútbol. Barcelona: Paidotribo.

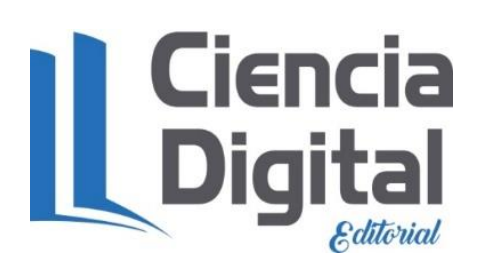




\section{PARA CITAR EL ARTÍCULO INDEXADO.}

Tapia Jara, F. G. (2021). Evolución de los sistemas de juego en el fútbol: Una revisión

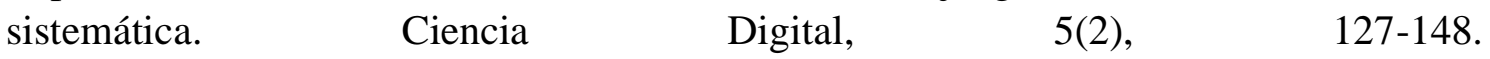
https://doi.org/10.33262/cienciadigital.v5i2.1620

\section{LCiencia}

El artículo que se publica es de exclusiva responsabilidad de los autores y no necesariamente reflejan el pensamiento de la Revista Ciencia Digital.

El artículo queda en propiedad de la revista y, por tanto, su publicación parcial y/o total en otro medio tiene que ser autorizado por el director de la Revista Ciencia Digital.
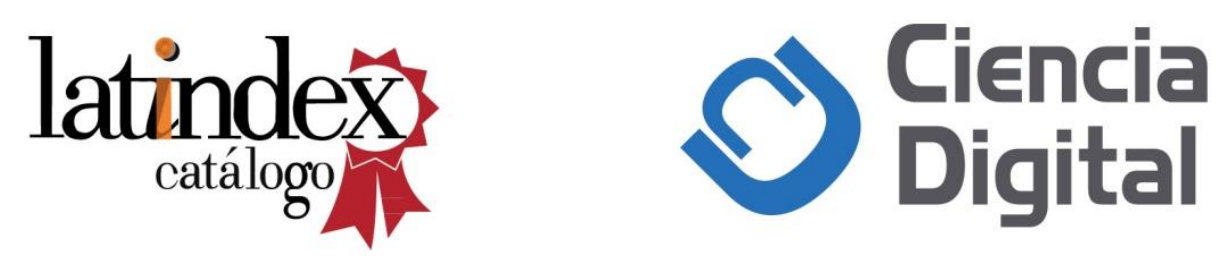